\title{
Kir4.1 may represent a novel therapeutic target for diabetic retinopathy (Review)
}

\author{
XIAOYU LI ${ }^{1,2}$, JIAJUN LV ${ }^{1,2}$, JIAZHI LI ${ }^{2}$ and XIANG REN ${ }^{1}$ \\ ${ }^{1}$ Department of Histology and Embryology, Dalian Medical University, Dalian, Liaoning 116044; ${ }^{2}$ Department of \\ Radiotherapy Oncology, The Second Hospital of Dalian Medical University, Dalian, Liaoning 116023, P.R. China
}

Received April 6, 2021; Accepted May 28, 2021

DOI: $10.3892 /$ etm.2021.10453

\begin{abstract}
As the major cause of irreversible loss of vision in adults, diabetic retinopathy (DR) is one of the most serious complications of diabetes. The imbalance of the retinal microenvironment and destruction of the blood-retinal barrier have a significant role in the progression of DR. Inward rectifying potassium channel 4.1 (Kir4.1) is located on Müller cells and is closely related to potassium homeostasis, water balance and glutamate clearance in the whole retina. The present review discusses the functions of Kir4.1 in regulating the retinal microenvironment and related biological mechanisms in DR. In the future, Kir4.1 may represent a novel alternative therapeutic target for DR through affecting the retinal microenvironment.
\end{abstract}

\section{Contents}

1. Introduction

2. Kir4.1-basics

3. Kir4.1 in the retina

4. Kir4.1 and the retinal microenvironment

5. Kir4.1 and related biological mechanisms in DR

6. Prospects

\section{Introduction}

Diabetic retinopathy (DR) is the most common serious complication of diabetes, mainly manifesting as progressive and irreversible vision damage. At present, there are 346 million

Correspondence to: Dr Jiazhi Li, Department of Radiotherapy Oncology, The Second Hospital of Dalian Medical University, 463 Zhong Shan Road, Dalian, Liaoning 116023, P.R. China

E-mail: jiazhilee@126.com

Dr Xiang Ren, Department of Histology and Embryology, Dalian Medical University, 9 Lvshun South Road West Section, Dalian, Liaoning 116044, P.R. China

E-mail: xiangren@dmu.edu.cn

Key words: Kir4.1, diabetic retinopathy, retinal microenvironment individuals with diabetes worldwide, $\sim 10 \%$ of which have severe visual impairment and $2 \%$ of them are blind. It is expected that the number of individuals at risk of vision loss from DR will be double by 2030 (1).

Strategies to prevent or treat DR early have become a research hotspot. An increasing number of studies have indicated that the occurrence of retinal neurodegenerative changes in DR may be earlier than microvascular changes. Furthermore, both the proliferation of glial cells and the damage of photoreceptor cells may occur at the beginning of the disease $(2,3)$. Most previous studies regarded DR as a vascular disease, but now comprehensive properties such as retinal neurodegeneration should also be considered (4). The pathogenesis of DR is highly complex. The changes in the microenvironment of the retina caused by oxidative stress, inflammatory response and other factors affect the occurrence and development of DR $(5,6)$.

The retinal microenvironment homeostasis mediated by inward rectifying potassium channel 4.1 (Kir4.1) on Müller cells is a basic system that provides the necessary conditions for retinal cells to maintain normal physiological functions. The balance of potassium ions, water and glutamic acid is adjusted through the bond of Kir4.1 (7). In DR, Kir4.1 expression is downregulated, Müller cells exhibit edema and apoptosis, and glutamate accumulates in the retinal microenvironment (8). This series of changes aggravates optical nerve damage. Based on the destruction of the retinal microenvironment, processes such as inflammation and oxidative stress occur in sequence to promote the progression of DR $(9,10)$. As the prime mediator of the entire retinal microenvironment regulation, Kir4.1 may be considered a novel target for the treatment of DR in the future.

\section{Kir4.1-basics}

The Kir channels facilitate potassium ions to enter cells and may be divided into four groups and seven Kir channel subfamilies according to their structure and function. Kir2.x is a classic Kir channel with constitutive activity. Kir3.x is a $G$ protein-gated Kir channel and Kir6.x is the adenosine triphosphate (ATP)-sensitive channel in cell metabolism. Furthermore, Kir1.x, Kir4.x, Kir5.x and Kir7.x are mainly responsible for the transportation of potassium ions (11).

Kir4.1 is a member of the Kir4.x family and is widely expressed in the nervous system. Kir4.1 is composed of 
four identical Kir4.1 subunits, which mediate potassium ion homeostasis, and water and glutamate transport inside and outside the cells (12). The Kir4.1 subunit is encoded by the KCNJ10 gene (13). Each subunit contains two transmembrane (TM) domains, TM-1 and TM-2. One of the extracellular loops contains the characteristic amino acid sequence $\mathrm{GYG}$, which is involved in the selective ion filtration of $\mathrm{K}^{+}$(14). Kir4.1 has been indicated to be associated with various conditions, including autism, epilepsy, central nervous system ischemic injury and inflammation (15). In these diseases, the expression of the Kir4.1 channel is reduced or misplaced and the continuous lack of Kir current damages the buffering capacity of glial cells, leading to the imbalance of ion homeostasis, which may contribute to pathological processes.

\section{Kir4.1 in the retina}

The expression of Kir4.1 varies among different tissues. In the retina, it is mainly distributed on Müller cells. Penetrating between the inner limiting membrane and the outer membrane of the retina, Müller cells are a type of astrocytes that are irreplaceable components of retinal cells. As they constitute the blood-retinal barrier, Müller cells are responsible for supporting the function and metabolism of peripheral neurons; they increase retinal compliance and resistance, secrete a variety of neuroprotective factors and participate in retinal angiogenesis (16-18). Kir4.1 is selectively enriched on the end feet of Müller cells surrounding microvessels and vitreous bodies, where the expression of Kir4.1 is significantly higher than that in other regions. It has been reported that $\beta 1$-syntrophin and Nogo-A may be involved in the polar expression of Kir4.1 on Müller cells $(19,20)$. Kir4.1 has a C-terminal sequence ending with-Ser-Asn-Val. This sequence may be recognized by $\beta 1$-syntrophin, which anchors Kir4.1 to the terminal feet of Müller cells and mediates the siphon effect of $\mathrm{K}^{+}$to maintain the $\mathrm{K}^{+}$steady state. The increase of Nogo-A expression does not lead to any increase in the total amount of Kir4.1 protein, but selectively affects Kir4.1 expression, causing it to redistribute on Müller cells, which in turn affects $\mathrm{K}^{+}$transport.

\section{Kir4.1 and the retinal microenvironment}

Overview. In the regulation of the retinal microenvironment, Kir4.1 maintains spatial potassium ion homeostasis, preserves the water balance and mediates glutamate transport. It is precisely due to the transport of $\mathrm{K}^{+}$that the osmotic pressure of the intracellular fluid of Müller cells changes accordingly and aquaporin 4 (AQP4) on the membrane of Müller cells thereby regulates the water balance and maintains the normal cell volume. Furthermore, the change of $\mathrm{K}^{+}$conductance also affects the electrochemical power of glutamate transport, enabling the function of glutamate/L-aspartate transporter (GLAST). The roles of Kir4.1 in the retinal microenvironment are presented in Fig. 1.

Kir4.1 and spatial stability of potassium ions. Kir4.1 moves large amounts of $\mathrm{K}^{+}$inwards at hyperpolarized potentials or potentials negative to the potassium equilibrium potential (EK). During the depolarization process, only a relatively small outward $\mathrm{K}^{+}$current may be observed, which is caused by the temporary blocking of the potassium ion channel by the positively charged polyamine and $\mathrm{Mg}^{2+}$. When the membrane potential is close to or more negative than the EK, these cations will move away from their binding site, allowing $\mathrm{K}^{+}$to flow through the channel, which is conducive to maintaining the cell's resting $\mathrm{K}^{+}$conductivity (21). However, the rectification function of Kir4.1 is weak and the current flowing into and out of the channel has a similar magnitude. Unlike Kir2.1, which is a strong inward rectifier potassium ion channel distributed on Müller cells (22), the weak rectifier characteristic of Kir4.1 may make Kir4.1 more susceptible to pathological conditions (23).

During neuron excitation, $\mathrm{K}^{+}$is released into the extracellular space. Increased extracellular $\mathrm{K}^{+}$promotes neuronal damage by triggering harmful secondary cascades. Sibille et al (24) determined that Kir4.1 is able to promote the fine-tuning of neuronal synapses, providing astrocytes with another effective mechanism for regulating neuronal activity. Their study further elucidated potassium dynamics between neurons, astrocytes and extracellular space according to a three-compartment model. In the retina, Kir4.1 has the most important role in maintaining the resting membrane potential and regulating the extracellular $\mathrm{K}^{+}$concentration to prevent the overexcitement of neurons (25). Specifically, Kir4.1 is able to transport excessive potassium ions in the microenvironment around the photoreceptor cells into the blood vessels or vitreous through its polar expression on Müller cells. Concurrently, Kir4.1 avoids excessive $\mathrm{K}^{+}$accumulation in Müller cells and high potassium load on the cells.

Kir4.1 and glutamate transport. Glutamate is the major excitatory neurotransmitter in the mammalian central nervous system and is involved in neural development, synaptic plasticity, and learning and memory function under physiological conditions. When its concentration is increased, it may cause neurotoxicity, resulting in neuron damage and degeneration (26). In order to prevent excessive glutamate levels outside of nerve cells, the body maintains normal glutamate levels through the glutamate receptor. Glutamate transporters may be divided into a variety of types, such as excitatory amino acid transporters (EAAT) and vesicle glutamate transporters, according to their structural and functional characteristics $(27,28)$. Glutamate/L-aspartate transporter (GLAST) is a subtype of EAAT, also known as EAAT1, mainly distributed on Müller cells in the retina (29). Excessive glutamate in the retina is mostly transferred to Müller cells through GLAST, where it is converted to glutamine by glutamine synthetase (GS) to be eliminated, reducing its neurotoxicity.

Glutamic acid is released from the presynaptic membrane and diffuses into the intersynaptic space due to an action potential, bringing about an increase in the extracellular glutamic acid concentration. GLAST on Müller cells requires energy to take up glutamate. The stochiometrics of the electrical generation process are as follows: $3 \mathrm{Na}^{+}, 1 \mathrm{H}^{+}$and 1 glutamic acid molecule enter the cell and $1 \mathrm{~K}^{+}$leaves the cell. Therefore, effective glutamate uptake requires not only the expression of functional glutamate uptake proteins but also a certain chemical gradient. The strong resting potential mediated by Kir4.1 generates an inward-directed electrochemical driving force, 


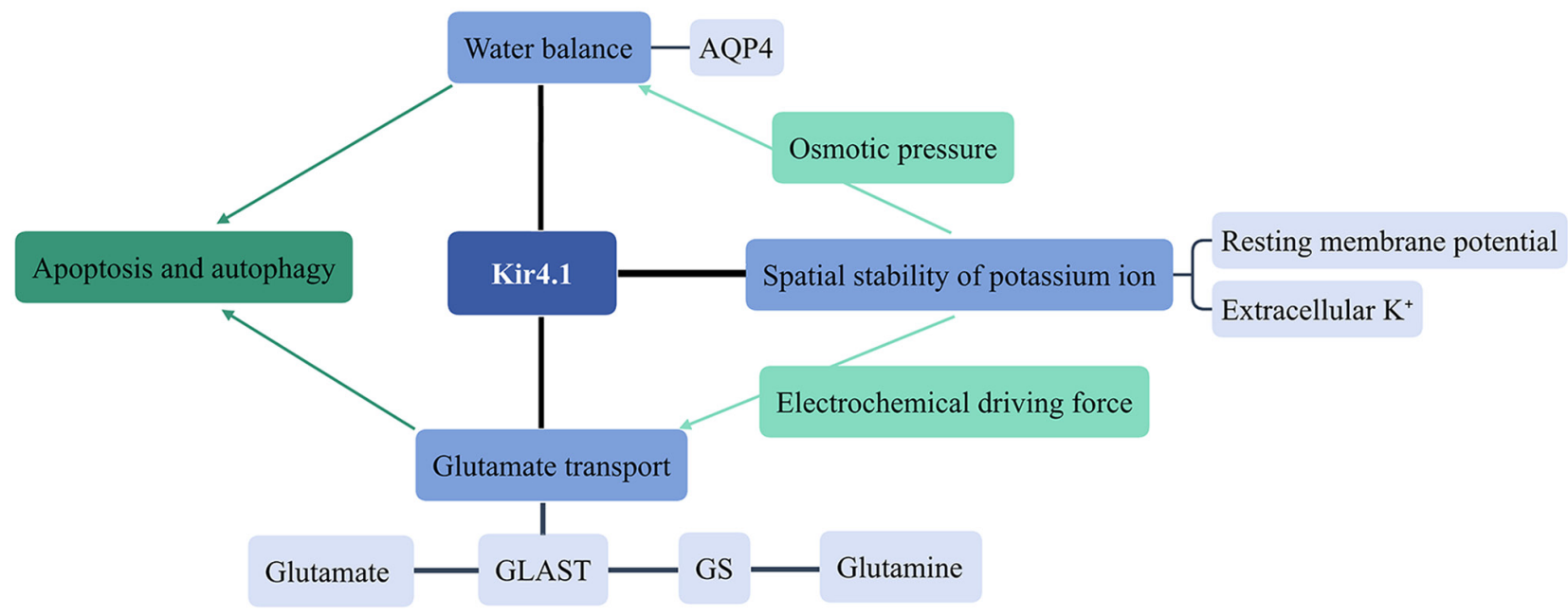

Figure 1. Kir4.1 and the retinal microenvironment. In the regulation of the retinal microenvironment, Kir4.1 maintains spatial potassium ion homeostasis, preserves the water balance and mediates glutamate transport. Kir4.1, inward rectifying potassium channel 4.1; AQP4, aquaporin 4; GLAST, glutamate/L-aspartate transporter; GS, glutamine synthetase.

giving GLAST the ability to promote the transfer of glutamate into Müller cells (30). The decrease of Kir4.1 channel activity causes the depolarization of the Müller cell's membrane, which weakens the driving force of the glutamate transporter that gives rise to the accumulation of extracellular glutamate and this inhibits GLAST-mediated glutamate transport (31). It was reported that pharmacological inhibition and small interfering RNA-mediated Kir4.1 knockdown in cortical astrocytes reduced glutamate uptake by 33.1 and $57.0 \%$, respectively (32).

Kir4.1 and water balance. The maintenance of water balance in the retina mainly depends on AQP4 expressed on the surface of Müller cells. Furthermore, Kir4.1 and AQP4 are co-localized in the perivascular region of astrocytes (33). The two have a certain coupling function. Due to the transport of $\mathrm{K}^{+}$, the osmotic pressure of Müller cells changes with the $\mathrm{K}^{+}$ concentration and the water balance is then adjusted by AQP4. Therefore, any abnormal $\mathrm{K}^{+}$channel function may lead to impaired retinal water clearance, which aggravates the development of retinal edema.

In particular, when Kir4.1 suffers functional impairment, the extracellular potassium ion concentration increases to cause depolarization of neurons and glia, which leads to increased glutamate efflux (34). At this stage, excessive potassium ions cannot be discharged and the accumulation in Müller cells causes the osmotic pressure to markedly increase. Glutamate-induced water inflow makes the swelling of Müller cells more severe, eventually leading to cell autophagy and apoptosis (35). As an important factor in maintaining the retinal microenvironment, apoptosis of Müller cells increases the severity of the imbalance of retinal water regulation, the damage of the spatial stability of potassium ions and the accumulation of glutamic acid, generating a vicious cycle.

\section{Kir4.1 and related biological mechanisms in DR}

Overview. Changes in the retinal microenvironment may affect inflammation, glutamate toxicity, oxidative stress, apoptosis, autophagy and biological rhythm. While knowledge regarding the direct involvement of Kir4.1 in DR remains limited, its roles in the associated mechanisms of inflammation, glutamate toxicity, oxidative stress, apoptosis, autophagy and biological rhythm may provide clues and they are discussed in the remainder of this chapter. The roles of Kir4.1 in biological mechanisms associated with DR are presented in Figs. 2 and 3.

Inflammation. A series of studies have suggested that DR is a chronic inflammatory lesion (36). Inflammatory changes in the retinal microenvironment are considered to be the main reason for the development of DR. It has been detected in both diabetic patients and animal models that the expression of AQP4 in the retina increases, Kir4.1 expression is downregulated and Müller cells are dysfunctional, which induces the destruction of the blood-retinal barrier (37-39).

Furthermore, it has been determined that Kir4.1 may cause edema of Müller cells by affecting the combined effect of osmotic pressure and AQP4, triggering the corresponding inflammatory response. As a key cause of visual impairment in diabetic patients, the occurrence of macular edema was previously thought to be due to vascular leakage resulting from the direct destruction of the blood-retinal barrier. However, failure of the active potassium transport mechanism mediated by Kir4.1 in the blood-retinal barrier may be a more important factor leading to diabetic macular edema of clinical significance (25). This change in the potassium channel may interfere with arachidonic acid-induced intracellular sodium overload, contributing to the rapid release of potassium ions and cell swelling (23). In addition to DR, AQP4 and Kir4.1 are involved in the inflammatory response of various neurological diseases such as ataxia, epilepsy and neurological deafness. Their local expression decrease or content increase leads to disturbance of the water and ion content of the glial cells. The volume of the cell and the structure of the adjacent tissue changes and the corresponding barrier is destroyed (40). After epileptic seizures, Kir4.1 transcription of astrocytes is downregulated and accompanied by upregulation of 


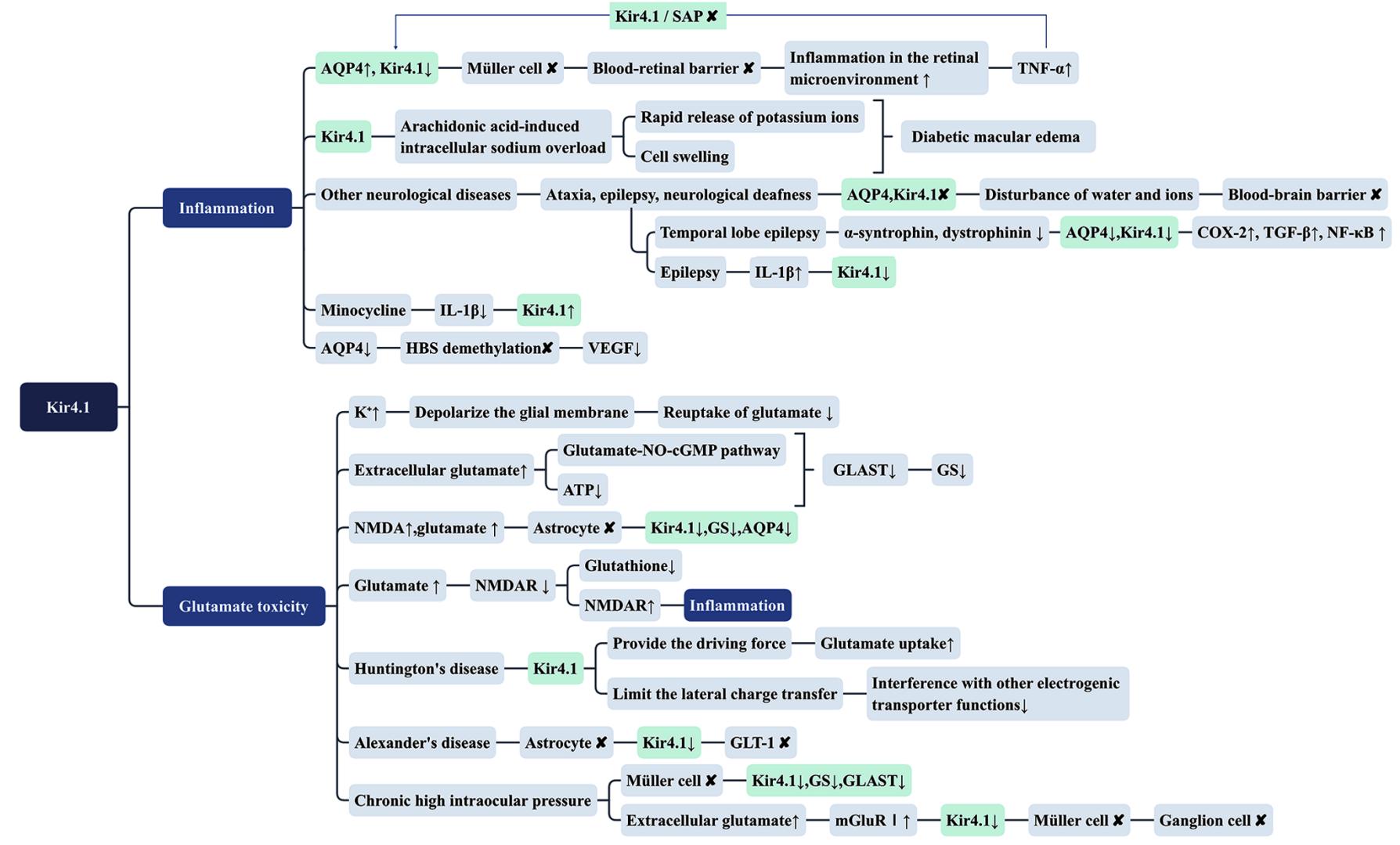

Figure 2. Kir4.1 and related biological mechanisms (inflammation and glutamate toxicity) in diabetic retinopathy. Kir4.1, inward rectifying potassium channel 4.1; AQP4, aquaporin 4; TNF- $\alpha$, tumor necrosis factor- $\alpha$; SAP, synapse-associated protein; COX-2, cyclooxygenase-2; HBS, hypoxia-inducible factor-1 binding site; NO, nitric oxide; cGMP, cyclic guanosine monophosphate; GLAST, glutamate/L-aspartate transporter; GS, glutamine synthetase; NMDA, N-methyl-D-aspartate; NMDAR, N-methyl-D-aspartate receptor; GLT-1, glutamate transporter-1; mGluRI, group I metabotropic glutamate receptor.

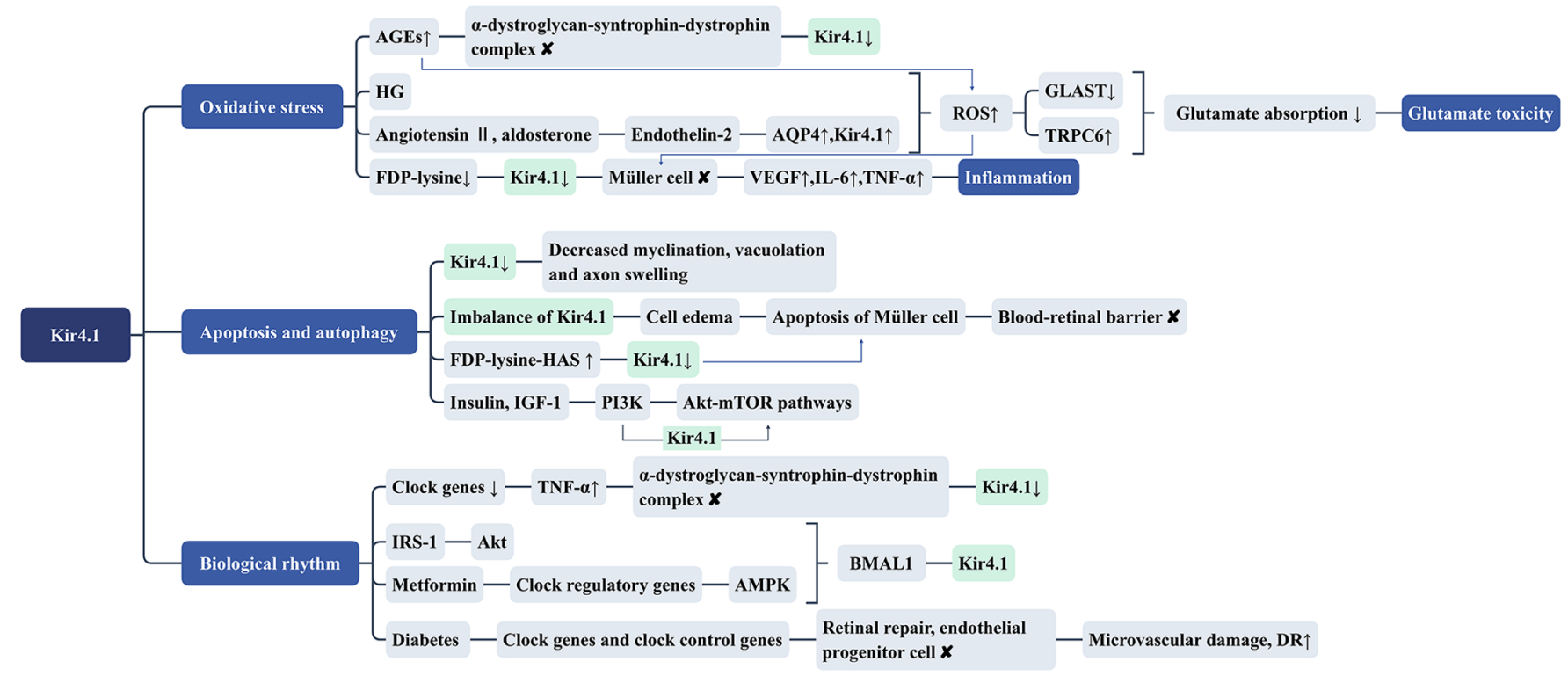

Figure 3. Kir4.1 and related biological mechanisms (oxidative stress, apoptosis and autophagy and biological rhythm) in DR. DR, diabetic retinopathy; Kir4.1, inward rectifying potassium channel 4.1; AGEs, advanced glycation end products; HG, high glucose; AQP4, aquaporin 4; ROS, reactive oxygen species; GLAST, glutamate/L-aspartate transporter; TRPC6, transient receptor potential cation channel 6; FDP-lysine, Ne-(3-formyl-3,4-dehydropiperidino)lysine; FDP-lysine-HAS, FDP-lysine-modified human serum albumin; IGF-1, insulin-like growth factor 1; IRS-1, insulin receptor substrate-1; AMPK, adenosine 5'-monophosphate-activated protein kinase; BMAL1, brain and muscle arnt-like protein 1.

various inflammatory genes (41). In temporal lobe epilepsy, Kir4.1 and AQP4 are downregulated simultaneously with $\alpha$-syntrophin and dystrophinin, while inflammatory factors, such as cyclooxygenase- 2 , TGF- $\beta$ and NF- $\mathrm{KB}$ significantly 
increase (42). The above studies confirmed again that Kir4.1 combined with AQP4 to cause edema of Müller cells and affect the osmotic pressure, leading to the destruction of the retinal barrier and promoting the subsequent release of inflammatory factors.

In addition, Kir4.1 and AQP4 may also be directly involved in the occurrence of inflammation through certain pathways, rather than indirectly affecting osmotic pressure. In certain diseases, AQP4 may promote the release of inflammatory factors through possible pathways $(43,44)$. A study indicated that upregulation of vascular endothelial growth factor (VEGF) during retinal hypoxia is related to the methylation status of the hypoxia-inducible factor-1 binding site (HBS). AQP4 deletion may prevent the upregulation of VEGF by interfering with the process of hypoxia-induced HBS demethylation (45). Whether Kir4.1 combines with AQP4 to directly promote inflammation in DR requires further research.

By contrast, inflammation may also act on Kir4.1. A study demonstrated that the expression changes of Kir4.1 in epilepsy-related lesions may be affected by the local inflammatory environment, particularly the inflammatory cytokine IL-1 $\beta$ (46). Another study reported that TNF- $\alpha$ is able to destroy the actin cytoskeleton and dissociation of Kir4.1/ synapse-associated protein (SAP) (47), indicating that inflammation destroys the attachment skeleton of Kir4.1. Another study suggested that treatment of the retina of diabetic rats with minocycline increased the level of Kir4.1 by reducing IL-1 $\beta$ levels (39). Therefore, inflammation may downregulate the polar expression of Kir4.1, leading to further deterioration of the retinal microenvironment. The specific mechanism remains to be elucidated.

Glutamate toxicity. In DR, downregulation of Kir4.1 reduces the ability of Müller cells to clear glutamate. High concentrations of potassium ions may reduce the binding force of potassium ions and depolarize the glial membrane, thereby reducing the reuptake of glutamate.

Increased extracellular glutamate concentrations may lead to decreased expression levels of GLAST and GS, resulting in abnormal synaptic transmission. This may be related to the glutamate-nitric oxide-cyclic guanosine monophosphate pathway and the reduction of ATP $(48,49)$. In addition, the expression of Kir4.1 may be mediated by the N-methyl-D-aspartate receptor (NMDAR) expressed on astrocytes. Continuous exposure of astrocytes to NMDA and Glu reduces the expression of Kir4.1, GS and AQP4 and also reduces the activity of GS (50). Excessive NMDAR activity may increase the incidence of central nervous system diseases related to glutamatergic tone and excitotoxicity (51). Due to the increased glutamate concentration in the protruding gap, NMDAR on astrocytes cannot drive the intracellular mechanism to exert a neuroprotective effect, resulting in a reduction in the supply of antioxidant glutathione induced by this mechanism, which further strengthens the toxic effect of glutamic acid (52). Furthermore, NMDAR on astrocytes is also able to increase the secretion of pro-inflammatory cytokines in cells stimulated by inflammation (53).

Similar observations were made in other neurodegenerative diseases. In Huntington's disease, glutamate uptake activity mainly depends on Kir4.1 (54). Glutamate transporter-1
(GLT-1) is an electron transporter that relies on the negative membrane potential provided by Kir4.1 to clear glutamate released from extracellular nerves. In Alexander's disease, Kir4.1 expression on astrocytes is reduced, GLT-1 function is impaired and glutamate toxicity occurs (55). Despite the limited research on the glutamate toxicity theory in DR in the past decades, it is undeniable that the disorder of glutamate transport by Kir4.1-mediated lack of electrochemical driving force may be one of the crucial foundations of retinal microenvironment destruction. One study indicated that chronic high intraocular pressure downregulated the expression of Kir4.1, GS and GLAST in Müller cells (56). Wang and Yang (57) reported that extracellular glutamate accumulation caused by high intraocular pressure activates group I metabotropic glutamate receptors on Müller cells, which induces the activation of Müller cells by downregulation of Kir4.1 and leads to apoptosis of ganglion cells. This change in the retinal microenvironment should not be limited to DR but is the basis of a variety of retinopathies.

Oxidative stress. In the development of DR, oxidative stress frequently affects the retinal microenvironment along with multiple mechanisms. Pannicke et al (23) tested the inhibitory effect of reducing agents on diabetic retina swelling, indicating that acute oxidative stress is related to the induction of glial cell swelling under anisotropic penetration conditions. Animal experiments suggested that the activation of enzymes producing various reactive oxygen species (ROS) and nitrogen species, as well as the mitochondrial pathway, are involved in the induction of osmotic swelling of Müller cells in the retinal tissues of transgenic rats (58). Furthermore, transient receptor potential cation channel 6 (TRPC6) is a $\mathrm{Ca}^{2+}$ permeable cation channel sensitive to oxidative stress, which is easily detected in Müller cells and highly expressed under High glucose (HG) conditions. HG increases the production of ROS, reduces the expression of GLAST and activates the TRPC6 channel, which initiates a reduction in glutamate absorption by Müller cells (29). Therefore, oxidative stress may affect the water balance and glutamate transport of Müller cells and they may have a close relationship with Kir4.1.

Studies have indicated that oxidative stress products may reduce Kir4.1 expression and interact with inflammation. The accumulation of $\mathrm{Ne}$-(3-formyl-3,4-dehydropiperidino)lysine (FDP-lysine, which is an oxidative stress lipid oxidation end product) causes Müller glial dysfunction with the protein level of the potassium channel subunit Kir4.1 decreasing and the downregulation of inwardly rectifying $\mathrm{K}^{+}$currents in Müller cells may, at least in part, be responsible for successive upregulation of VEGF, IL-6 and TNF- $\alpha$ (59). Endothelin-2 is a potent vasoconstrictor. Angiotensin II and aldosterone stimulate endothelin-2 expression, which promotes Müller glial dysfunction and blood-retinal barrier destruction by generating ROS. In this process, endothelin-2 was determined to increase AQP4 and Kir4.1 in Müller cells and their mRNA levels in the retina (60). Apart from this, the accumulation of advanced glycation end products (AGEs) is the major pathological event in DR. AGEs promote the formation of ROS, which boost the production of AGEs, resulting in positive feedback loops that compromise tissue fitness (61). AGE-modified laminin causes disorder of the actin cytoskeleton and destruction of 
the $\alpha$-dystroglycan-syntrophin-dystrophin complex, which in turn reduces Kir4.1 expression and weakens its function (62). Evidently, oxidative stress is part of various complex mechanisms involved in Kir4.1.

Apoptosis and autophagy. Autophagy and apoptosis frequently occur as intermediate processes in the development of DR, which are a result of the combination of various factors such as inflammation, oxidative stress, cellular edema and glutamate toxicity. In other nerve tissues, there is evidence that Kir4.1 is involved in apoptosis. The loss of Kir4.1 results in decreased myelination, vacuolation and axon swelling in the spinal cord of mice, which are all related to apoptosis (63). Kir4.1 expressed on Müller cells allows neurons that generate action potentials in the inner layer of the retina to maintain their function in a potassium buffer environment, particularly under ischemic conditions. Maintaining the function of Müller cells may suppress cytotoxic neuron overexcitation and subsequent neuronal cell loss (64). The cell edema caused by the imbalance of Kir4.1 in DR may trigger apoptosis of Müller cells, destroy the retinal barrier and eventually lead to irreparable visual impairment.

High concentrations of FDP-lysine-modified human serum albumin cause extensive apoptosis. The accumulation of these adducts may lead to the apoptosis of Müller cells during long-term diabetes, while the accumulation of FDP-lysine is associated with a reduction in the level of potassium channel subunit Kir4.1 protein (59). Therefore, Kir4.1 may be related to cell apoptosis. Furthermore, Kir4.1 may prevent the induction of autophagy via phosphoinositide 3-kinase (PI3K)-dependent activation through the mechanistic target of rapamycin (mTOR) pathway. Akt is an important factor involved in cell survival and apoptosis and mTOR is a downstream target of Akt. After ischemia and hypoxia, the level of extracellular potassium ions increases significantly. Subsequently, Akt is also phosphorylated by recruiting $\mathrm{PI} 3 \mathrm{~K}$ to activate $\mathrm{Kir} 4.1$, which triggers the mTOR pathway to enable cell survival (65). One study has indicated that the heteromer Kir4.1/Kir5.1 channel in the renal cortical collecting duct may be activated in a PI3K-dependent manner by insulin and insulin-like growth factor-1, indicating that Kir4.1 is likely to interact with the Akt pathway (66). Despite the limited evidence available, increasing the activity of Akt may become a novel strategy to prevent DR. Numerous studies have indicated that activation of Akt may protect nerve cells from oxidative stress and inhibition of the function of PTEN has considerable pharmacological value in ischemia-related diseases and neurodegenerative diseases such as Alzheimer's disease and Parkinson's disease (67). In summary, the increased expression and activity of Kir4.1 may effectively antagonize the autophagy and apoptosis mediated by various mechanisms in DR.

Biological rhythm. Biological rhythms are not only able to regulate the sleep-wake cycle of an organism but are also related to the secretion and release of hormones. Among the core genes relevant to biological rhythms, the clock circadian regulator gene, brain and muscle aryl hydrocarbon receptor nuclear translocator-like 1 gene (BMAL1, also known as ARNTL) and period circadian clock gene have gained widespread attention. A series of recent studies suggested that biological rhythms have a certain impact on diabetes, mainly through metabolism $(68,69)$. The vision of patients with diabetes changes regularly with day and night and their electroretinograms also indicate that the $a$ and $b$ waves of the retina have a biological rhythm (70).

The expression of Kir4.1 in the retina also exhibits specific biological rhythms. Disturbances in the daily expression pattern of clock genes and clock control genes caused by diabetes may lead to loss of synchronization of retinal repair as well as the release and migration of endothelial progenitor cells required for effective repair processes, contributing to increased microvascular damage and DR (71). Hassan et al (47) indicated that silencing of clock genes upregulated the expression of TNF- $\alpha$, leading to the destruction of the actin cytoskeleton and dissociation of Kir4.1/SAP. Another study reported that insulin receptor substrate-1 (IRS-1) is able to regulate Kir4.1 on Müller cells through the BMAL1 gene and IRS-1-mediated dysfunction signals may downregulate Kir4.1 expression. IRS-1 blockade indirectly inhibits Kir4.1 by downregulating the IRS1-Akt-BMAL1 axis and the Kir4.1 rhythm is synchronized with insulin-mediated signaling (72). Metformin, as a traditional hypoglycemic agent, may indirectly activate 5'adenosine monophosphate (AMP)-activated protein kinase (AMPK) by inhibiting the mitochondrial complex (73). A recent animal study indicated that metformin may increase the expression of BMAL1 protein and Kir4.1 by controlling clock regulatory genes to activate the AMPK pathway (74). For the retina with considerable circulation, the expression of Kir4.1 is regulated by the BMAL1 gene through the influence of light and dark cycles, which has a close effect on retinal metabolism and inflammation. The circadian rhythm of Kir4.1 is synchronized with the rhythm of IRS-1 and the tyrosine phosphorylation of IRS-1 provides binding sites for multiple signaling molecules, particularly the activation of Akt. At the same time, Akt activation involves mechanisms such as cell survival, metabolism and apoptosis. AMPK is a sensor of energy availability within the cell, which is activated at low energy levels and regulates cellular processes accordingly. In addition, AMPK activity supports systemic glucose homeostasis and improves insulin sensitivity by promoting processes such as glucose uptake and energy expenditure. Glucose deficiency, hypoxia, oxidative stress and other changes may reduce cell energy levels and increase the AMP/ATP ratio, thereby activating AMPK. Activated AMPK is able to indirectly inhibit the mechanistic target of rapamycin complex 1 (mTORC1) by counteracting the inhibitory effects of mTORC1 and IRS-1 (75). In addition, the inflammatory environment may be involved in influencing IRS-1-Kir4.1 signaling. Since biological rhythms may interact with various mechanisms such as autophagy and oxidative stress to affect Kir4.1, Akt and AMPK may be important targets for drug design to prevent Müller cell dysfunction in DR.

\section{Prospects}

DR is a chronic inflammatory neurodegenerative disease with multiple mechanisms, which ultimately damages vision and even leads to permanent loss of sight. This type of vision impairment is irreversible. In the early stages of DR, the major pathological manifestations are changes in microvascular 
function, including retinal vasodilation and microaneurysms, while in the later stage, vitreous integrity is lost, new blood vessels are ruptured and bleed and normal physiological functions of the retina are lost (76). However, the current clinical treatment of DR mostly focuses on the period of vascular hyperplasia with obvious symptoms, which cannot reverse the loss of vision. Therefore, it is necessary to explore a path for early prevention and treatment of DR.

To pursue the new therapeutic direction involving Kir4.1, its molecular biological mechanisms require to be thoroughly investigated. There are three approaches worth exploring, as discussed below: i) Containment of inflammatory response; ii) in-depth study of Akt- and AMPK-targeted treatments; and iii) further exploration of therapies targeting the Kir4.1 cytoskeleton.

DR is a chronic inflammatory disease and the core part of the treatment lies in exerting anti-inflammatory effects in the retinal microenvironment. In DR, disorder of Kir4.1 induces changes in the retinal microenvironment, accompanied by upregulation of inflammatory factors. Inflammation and oxidative stress products destroy the attachment framework of Kir4.1 to cause further imbalance, aggravating the destruction of the retinal microenvironment. It is necessary to further explore the specific pathways of the arachidonic acid pathway that Kir4.1 may participate in. Furthermore, certain inflammation-related factors are also worthy of attention from researchers, particularly VEGF. Various clinical trials have proved that anti-VEGF treatment in the vitreous is an important therapeutic method for diabetic macular edema (77). VEGF is an important mediator of the inflammation release pathway and AQP4 has been indicated to have a regulatory relationship with VEGF. Kir4.1 may also participate in this process together with AQP4.

Akt and AMPK may be targets for DR treatment. On the one hand, PI3K-Akt-mTOR is a classic pathway that responds to insulin signaling. The Kir4.1 rhythm is synchronized with insulin-mediated signaling and Kir4.1 may activate Akt indirectly in a PI3K-dependent manner. Furthermore, the PI3K/ Akt pathway may be regulated by GluN2A subunit-containing NMDAR and the expression of Kir4.1 may be related to NMDAR (78). A number of animal experiments have indicated that NMDAR inhibitors are able to reduce the death of retinal neurons in DR caused by glutamate toxicity (79-81). The design of drugs targeting Kir4.1 to activate Akt may be a novel treatment approach, but this is required to be verified by further research. On the other hand, AMPK is also crucial for the survival of Müller cells. In the high-glucose and hypoxia environment of DR, ensuring sufficient levels of Müller cells is needed in order to maintain the integrity of the blood-eye barrier, which may enhance the stability of the retinal microenvironment (82-84). A previous study has indicated that AMPK activation protects astrocytes from hypoxia-induced cell death (85). Upregulation of Kir4.1 is related to the activation of the AMPK pathway. Furthermore, a novel study in the field of biological rhythms has indicated that therapies targeting IRS-1-Kir4.1 signaling may be of great clinical significance (72).

Protecting the normal structure of Kir4.1's attached cytoskeleton may become another novel therapeutic aspect. Improving the dislocation of Kir4.1 may have a more important role than increasing its expression level. A study indicated that proliferative gliosis in the retina is related to the inactivation of glial Kir channels, which is not caused by the downregulation of channel proteins, but is related to their dislocation in the cell membrane (86). Dystrophin (Dp)71 is a membrane-associated cytoskeletal protein on Müller cells, which is the core anchor for the polar expression of AQP4 and Kir4.1 (87). From the perspective of membrane cytoskeletal proteins, a study has indicated that $\mathrm{AAV}$-mediated gene therapy has an effect on the blood-retinal barrier of dystrophin-Dp71-deficient mice. The distribution of Kir4.1 and AQP4 has a restorative effect to ameliorate the condition of retinal edema (88). It has also been reported that the adrenal cortex hormone dexamethasone is able to inhibit the downregulation of Dp71 by inhibiting the downregulation of heat shock factor 1 , thereby inhibiting the downregulation of AQP4 and Kir4.1 in Müller cells to prevent the occurrence and development of blood-retinal barrier destruction (89).

Furthermore, Kir4.1 and AQP4 are related to each other in positional expression and function and they may have a synergistic effect via related mechanisms. However, at present, relevant studies are limited. In the future, the mechanisms mediated by Kir4.1 and AQP4 will become the focus of research. However, it is worth noting that Kir4.1 and AQP4 are not completely coupled. One study indicated that Kir4.1 and AQP4 clusters were co-localized in the perivascular area, but not in parenchyma (33). An animal experiment indicated that after intravitreal injection of lipopolysaccharide during rat uveitis, the expression of Kir4.1 in the retina decreased significantly at the protein and gene levels, but the expression of AQP4 remained almost unchanged (90). Evidently, the relationship between Kir4.1 and AQP4 is exceedingly complex and further research still needs a long way to go.

Kir4.1-targeted treatment has been effectively explored, although the relevant mechanisms have remained to be elucidated. Sun et al (91) observed in rat model of DR that after the application of the potassium channel opener pinadil, the expression of Kir4.1 was upregulated, the function of Müller cells was improved and the symptoms of retinal macular edema were alleviated. Another study suggested that aloe vera may help protect from retinal damage associated with liver failure by normalizing Kir4.1 and AQP4 through reducing oxidative stress and inflammation (92).

In conclusion, Kir4.1 is likely to represent a novel alternative therapeutic target for DR through affecting the retinal microenvironment. It is thought that the mechanisms of Kir4.1 will be ulteriorly clarified with the deepening of future research.

\section{Acknowledgements}

Not applicable.

\section{Funding}

This work was supported by the Natural Science Foundation of Liaoning Province (grant no. 2020-BS-189), the National Natural Science Foundation of China (grant no. 31371218), the Basic Scientific Research Projects of Liaoning Provincial Education Department (grant no. LQ2017005) and the 
Liaoning Provincial Program for Top Discipline of Basic Medical Sciences (grant nos. 2020-BS-189 and LQ2017005).

\section{Availability of data and materials}

Not applicable.

\section{Authors' contributions}

XYL conceived the topic of the review. XYL and JJL retrieved and analyzed relevant documents. JJL was responsible for drawing the figures. XYL wrote the manuscript. JZL and XR revised the manuscript. All authors read and approved the final manuscript. Data sharing is not applicable.

\section{Ethics approval and consent to participate}

Not applicable.

\section{Patient consent for publication}

Not applicable.

\section{Competing interests}

The authors declare that they have no competing interests.

\section{References}

1. Mohammad HMF, Sami MM, Makary S, Toraih EA, Mohamed AO and El-Ghaiesh SH: Neuroprotective effect of levetiracetam in mouse diabetic retinopathy: Effect on glucose transporter-1 and GAP43 expression. Life Sci 232: 116588, 2019.

2. Hafner J, Zadrazil M, Grisold A, Ricken G, Krenn M, Kitzmantl D, Pollreisz A, Gleiss A and Schmidt-Erfurth U: Retinal and corneal Neurodegeneration and their association with systemic signs of peripheral neuropathy in type 2 diabetes. Am J Ophthalmol 209: 197-205, 2020.

3. Lynch SK and Abramoff MD: Diabetic retinopathy is a neurodegenerative disorder. Vision Res 139: 101-107, 2017.

4. Wang W and Lo ACY: Diabetic retinopathy: Pathophysiology and treatments. Int J Mol Sci 19: 1816, 2018.

5. Dehdashtian E, Mehrzadi S, Yousefi B, Hosseinzadeh A, Reiter RJ, Safa M, Ghaznavi H and Naseripour M: Diabetic retinopathy pathogenesis and the ameliorating effects of melatonin; involvement of autophagy, inflammation and oxidative stress. Life Sci 193: 20-33, 2018.

6. He M, Long P, Guo L, Zhang M, Wang S and He H: Fushiming capsule attenuates diabetic rat retina damage via antioxidation and anti-inflammation. Evid Based Complement Alternat Med 2019: 5376439, 2019.

7. Noël G, Belda M, Guadagno E, Micoud J, Klöcker N and Moukhles H: Dystroglycan and Kir4.1 coclustering in retinal Müller glia is regulated by laminin-1 and requires the PDZ-ligand domain of Kir4.1. J Neurochem 94: 691-702, 2005.

8. Coughlin BA, Feenstra DJ and Mohr S: Müller cells and diabetic retinopathy. Vision Res 139: 93-100, 2017.

9. Curtis TM, Hamilton R, Yong PH, McVicar CM, Berner A, Pringle R, Uchida K, Nagai R, Brockbank S and Stitt AW: Müller glial dysfunction during diabetic retinopathy in rats is linked to accumulation of advanced glycation end-products and advanced lipoxidation end-products. Diabetologia 54: 690-698, 2011.

10. Bringmann A, Pannicke T, Grosche J, Francke M, Wiedemann P, Skatchkov SN, Osborne NN and Reichenbach A: Müller cells in the healthy and diseased retina. Prog Retin Eye Res 25: 397-424, 2006.

11. Hibino H, Inanobe A, Furutani K, Murakami S, Findlay I and Kurachi Y: Inwardly rectifying potassium channels: Their structure, function, and physiological roles. Physiol Rev 90: 291-366, 2010 .
12. Mendez-Gonzalez MP, Kucheryavykh YV, Zayas-Santiago A Vélez-Carrasco W, Maldonado-Martínez G, Cubano LA, Nichols CG, Skatchkov SN and Eaton MJ: Novel KCNJ10 gene variations compromise function of inwardly rectifying potassium channel 4.1. J Biol Chem 291: 7716-7726, 2016.

13. Nwaobi SE and Olsen ML: Correlating Gene-specific DNA methylation changes with expression and transcriptional activity of astrocytic KCNJ10 (Kir4.1). J Vis Exp: 52406, 2015.

14. Ohno Y, Kinboshi M and Shimizu S: Inwardly rectifying potassium channel Kir4.1 as a novel modulator of BDNF expression in astrocytes. Int J Mol Sci 19: 3313, 2018.

15. Thuringer D, Chanteloup G, Boucher J, Pernet N, Boudesco C, Jego G, Chatelier A, Bois P, Gobbo J, Cronier L, et al: Modulation of the inwardly rectifying potassium channel Kir4.1 by the pro-invasive miR-5096 in glioblastoma cells. Oncotarget 8: 37681-37693, 2017

16. Govetto A, Hubschman JP, Sarraf D, Figueroa MS, Bottoni F, dell'Omo R, Curcio CA, Seidenari P, Delledonne G, Gunzenhauser $\mathrm{R}$, et al: The role of Müller cells in tractional macular disorders: An optical coherence tomography study and physical model of mechanical force transmission. Br J Ophthalmol 104: 466-472, 2020.

17. Eastlake K, Luis J and Limb GA: Potential of Müller glia for retina neuroprotection. Curr Eye Res 45: 339-348, 2020.

18. Li X, Liu J, Hoh J and Liu J: Müller cells in pathological retinal angiogenesis. Transl Res 207: 96-106, 2019.

19. Rao SB, Katoozi S, Skauli N, Froehner SC, Ottersen OP, Adams ME and Amiry-Moghaddam M: Targeted deletion of 31-syntrophin causes a loss of Kir 4.1 from Müller cell endfeet in mouse retina. Glia 67: 1138-1149, 2019.

20. Joly S, Dodd DA, Grewe BF and Pernet V: Reticulon 4A/Nogo-A influences the distribution of Kir4.1 but is not essential for potassium conductance in retinal Müller glia. Neurosci Lett 627: 168-177, 2016.

21. Nwaobi SE, Cuddapah VA, Patterson KC, Randolph AC and Olsen ML: The role of glial-specific Kir4.1 in normal and pathological states of the CNS. Acta Neuropathol 132: 1-21, 2016.

22. Kofuji P, Biedermann B, Siddharthan V, Raap M, Iandiev I, Milenkovic I, Thomzig A, Veh RW, Bringmann A and Reichenbach A: Kir potassium channel subunit expression in retinal glial cells: Implications for spatial potassium buffering. Glia 39: 292-303, 2002.

23. Pannicke T, Iandiev I, Wurm A, Uckermann O, vom Hagen F, Reichenbach A, Wiedemann P, Hammes HP and Bringmann A: Diabetes alters osmotic swelling characteristics and membrane conductance of glial cells in rat retina. Diabetes 55: 633-639, 2006.

24. Sibille J, Dao Duc K, Holcman D and Rouach N: The neuroglial potassium cycle during neurotransmission: Role of Kir4.1 channels. PLoS Comput Biol 11: e1004137, 2015.

25. Mori F, Hikichi T, Takahashi J, Nagaoka T and Yoshida A: Dysfunction of active transport of blood-retinal barrier in patients with clinically significant macular edema in type 2 diabetes. Diabetes Care 25: 1248-1249, 2002.

26. Wang Y and Qin ZH: Molecular and cellular mechanisms of excitotoxic neuronal death. Apoptosis 15: 1382-1402, 2010.

27. Li F, Eriksen J, Finer-Moore J, Chang R, Nguyen P, Bowen A, Myasnikov A, Yu Z, Bulkley D, Cheng Y, et al: Ion transport and regulation in a synaptic vesicle glutamate transporter. Science 368: 893-897, 2020.

28. Pavić A, Holmes AOM, Postis VLG and Goldman A: Glutamate transporters: A broad review of the most recent archaeal and human structures. Biochem Soc Trans 47: 1197-1207, 2019.

29. Ma M, Zhao S, Zhang J, Sun T, Fan Y and Zheng Z: High glucose-induced TRPC6 channel activation decreases glutamate uptake in rat retinal Müller cells. Front Pharmacol 10: 1668, 2019.

30. Kharade SV, Kurata H, Bender AM, Blobaum AL, Figueroa EE, Duran A, Kramer M, Days E, Vinson P, Flores D, et al: Discovery, characterization, and effects on renal fluid and electrolyte excretion of the Kir4.1 potassium channel pore blocker, VU0134992. Mol Pharmacol 94: 926-937, 2018.

31. Frizzo ME: Can a selective serotonin reuptake inhibitor act as a glutamatergic modulator? Curr Ther Res Clin Exp 87: 9-12, 2017.

32. Kucheryavykh YV, Kucheryavykh LY, Nichols CG, Maldonado HM, Baksi K, Reichenbach A, Skatchkov SN and Eaton MJ: Downregulation of Kir4.1 inward rectifying potassium channel subunits by RNAi impairs potassium transfer and glutamate uptake by cultured cortical astrocytes. Glia 55: 274-281, 2007. 
33. Smith AJ and Verkman AS: Superresolution imaging of Aquaporin-4 cluster size in antibody-stained paraffin brain sections. Biophys J 109: 2511-2522, 2015.

34. Djukic B, Casper KB, Philpot BD, Chin LS and McCarthy KD: Conditional knock-out of Kir4.1 leads to glial membrane depolarization, inhibition of potassium and glutamate uptake, and enhanced short-term synaptic potentiation. J Neurosci 27 11354-11365, 2007.

35. Reichenbach A and Bringmann A: New functions of Müller cells. Glia 61: 651-678, 2013.

36. Rübsam A, Parikh S and Fort PE: Role of inflammation in diabetic retinopathy. Int J Mol Sci 19: 942, 2018.

37. Vujosevic S, Micera A, Bini S, Berton M, Esposito G and Midena E: Aqueous humor biomarkers of Müller cell activation in diabetic eyes. Invest Ophthalmol Vis Sci 56: 3913-3918, 2015.

38. Li XM, Wendu RL, Yao J, Ren Y, Zhao YX, Cao GF, Qin J and Yan B: Abnormal glutamate metabolism in the retina of aquaporin 4 (AQP4) knockout mice upon light damage. Neurol Sci 35: 847-853, 2014.

39. Zhang Y, Xu G, Ling Q and Da C: Expression of aquaporin 4 and Kir4.1 in diabetic rat retina: Treatment with minocycline. J Int Med Res 39: 464-479, 2011.

40. Setkowicz Z, Kosonowska E and Janeczko K: Inflammation in the developing rat modulates astroglial reactivity to seizures in the mature brain. J Anat 231: 366-379, 2017.

41. Frigerio F, Frasca A, Weissberg I, Parrella S, Friedman A, Vezzani A and Noé FM: Long-lasting pro-ictogenic effects induced in vivo by rat brain exposure to serum albumin in the absence of concomitant pathology. Epilepsia 53: 1887-1897, 2012.

42. Das A, Wallace GC IV, Holmes C, McDowell ML, Smith JA, Marshall JD, Bonilha L, Edwards JC, Glazier SS, Ray SK and Banik NL: Hippocampal tissue of patients with refractory temporal lobe epilepsy is associated with astrocyte activation, inflammation, and altered expression of channels and receptors. Neuroscience 220: 237-246, 2012.

43. Wu J, Ding D, Wang X, Li Q, Sun Y, Li L and Wang Y: Regulation of aquaporin 4 expression by lipoxin A4 in astrocytes stimulated by lipopolysaccharide. Cell Immunol 344: 103959, 2019.

44. Li Y, Lu H, Lv X, Tang Q, Li W, Zhu H and Long Y: Blockade of aquaporin 4 inhibits irradiation-induced pulmonary inflammation and modulates macrophage polarization in mice. Inflammation 41: 2196-2205, 2018.

45. Pisani F, Cammalleri M, Dal Monte M, Locri F, Mola MG, Nicchia GP, Frigeri A, Bagnoli P and Svelto M: Potential role of the methylation of VEGF gene promoter in response to hypoxia in oxygen-induced retinopathy: Beneficial effect of the absence of AQP4. J Cell Mol Med 22: 613-627, 2018.

46. Zurolo E, de Groot M, Iyer A, Anink J, van Vliet EA, Heimans JJ, Reijneveld JC, Gorter JA and Aronica E: Regulation of Kir4.1 expression in astrocytes and astrocytic tumors: A role for interleukin-1 $\beta$. J Neuroinflammation 9: 280, 2012.

47. Hassan I, Luo Q, Majumdar S, Dominguez JM II, Busik JV and Bhatwadekar AD: Tumor necrosis factor Alpha (TNF- $\alpha$ ) disrupts Kir4.1 channel expression resulting in Müller cell dysfunction in the retina. Invest Ophthalmol Vis Sci 58: 2473-2482, 2017.

48. Lin Z, Huang P, Huang S, Guo L, Xu X, Shen X, Xie B and Zhong Y: Effect of adenosine and adenosine receptor antagonists on retinal Müller cell inwardly rectifying potassium channels under exogenous glutamate stimulation. Biomed Res Int 2018 2749257, 2018.

49. Saeed Dar M: Functional role for mouse cerebellar NO/cGMP/ KATP pathway in ethanol-induced ataxia. Alcohol Clin Exp Res 38: 100-107, 2014

50. Skowrońska K, Obara-Michlewska M, Zielińska $\mathrm{M}$ and Albrecht J: NMDA receptors in astrocytes: In search for roles in neurotransmission and astrocytic homeostasis. Int J Mol Sci 20: 309, 2019.

51. Gonzalez J, Jurado-Coronel JC, Ávila MF, Sabogal A, Capani F and Barreto GE: NMDARs in neurological diseases: A potential therapeutic target. Int J Neurosci 125: 315-327, 2015.

52. Jimenez-Blasco D, Santofimia-Castaño P, Gonzalez A, Almeida A and Bolaños JP: Astrocyte NMDA receptors' activity sustains neuronal survival through a Cdk5-Nrf2 pathway. Cell Death Differ 22: 1877-1889, 2015.

53. Skowrońska K, Obara-Michlewska M, Czarnecka A, Dąbrowska K, Zielińska M and Albrecht J: Persistent overexposure to N-Methyl-D-Aspartate (NMDA) calcium-dependently downregulates glutamine synthetase, aquaporin 4, and Kir4.1 channel in mouse cortical astrocytes. Neurotox Res 35: 271-280, 2019.
54. Dvorzhak A, Vagner T, Kirmse K and Grantyn R: Functional indicators of glutamate transport in single striatal astrocytes and the influence of Kir4.1 in normal and huntington mice. J Neurosci 36: 4959-4975, 2016.

55. Minkel HR, Anwer TZ, Arps KM, Brenner M and Olsen ML: Elevated GFAP induces astrocyte dysfunction in caudal brain regions: A potential mechanism for hindbrain involved symptoms in type II Alexander disease. Glia 63: 2285-2297, 2015.

56. Yang Z, Huang P, Liu X, Huang S, Deng L, Jin Z, Xu S, Shen X, Luo $\mathrm{X}$ and Zhong Y: Effect of adenosine and adenosine receptor antagonist on Müller cell potassium channel in Rat chronic ocular hypertension models. Sci Rep 5: 11294, 2015.

57. Wang ZF and Yang XL: Glutamate receptor-mediated retinal neuronal injury in experimental glaucoma. Sheng Li Xue Bao 68: 483-491, 2016 (In Chinese).

58. Vogler S, Pannicke T, Hollborn M, Grosche A, Busch S, Hoffmann S, Wiedemann P, Reichenbach A, Hammes HP and Bringmann A: Müller cell reactivity in response to photoreceptor degeneration in rats with defective polycystin-2. PLoS One 8: e61631, 2014

59. Yong PH, Zong H, Medina RJ, Limb GA, Uchida K, Stitt AW and Curtis TM: Evidence supporting a role for $\mathrm{N}$-(3-formyl-3,4-dehydropiperidino)lysine accumulation in Müller glia dysfunction and death in diabetic retinopathy. Mol Vis 16: 2524-2538, 2010.

60. Alrashdi SF, Deliyanti D, Talia DM and Wilkinson-Berka JL: Endothelin-2 injures the blood-retinal barrier and macroglial Müller cells: Interactions with angiotensin ii, aldosterone, and NADPH oxidase. Am J Pathol 188: 805-817, 2018.

61. Aragonès G, Rowan S, G Francisco S, Yang W, Weinberg J, Taylor A and Bejarano E: Glyoxalase system as a therapeutic target against diabetic retinopathy. Antioxidants (Basel) 9: 1062, 2020.

62. Thompson K, Chen J, Luo Q, Xiao Y, Cummins TR and Bhatwadekar AD: Advanced glycation end (AGE) product modification of laminin downregulates Kir4.1 in retinal Müller cells. PLoS One 13: e0193280, 2018.

63. Neusch C, Rozengurt N, Jacobs RE, Lester HA and Kofuji P: Kir4.1 potassium channel subunit is crucial for oligodendrocyte development and in vivo myelination. J Neurosci 21: 5429-5438, 2001.

64. Pannicke T, Frommherz I, Biedermann B, Wagner L, Sauer K, Ulbricht E, Härtig W, Krügel U, Ueberham U, Arendt T, et al: Differential effects of P2Y1 deletion on glial activation and survival of photoreceptors and amacrine cells in the ischemic mouse retina. Cell Death Dis 5: e1353, 2014.

65. Milton M and Smith PD: It's all about timing: The involvement of Kir4.1 channel regulation in acute ischemic stroke pathology. Front Cell Neurosci 12: 36, 2018

66. Zaika O, Palygin O, Tomilin V, Mamenko M, Staruschenko A and Pochynyuk O: Insulin and IGF-1 activate Kir4.1/5.1 channels in cortical collecting duct principal cells to control basolateral membrane voltage. Am J Physiol Renal Physiol 310: F311-F321, 2016.

67. Nitulescu GM, Van De Venter M, Nitulescu G, Ungurianu A, Juzenas P, Peng Q, Olaru OT, Grădinaru D, Tsatsakis A, Tsoukalas $\mathrm{D}$, et al: The Akt pathway in oncology therapy and beyond (Review). Int J Oncol 53: 2319-2331, 2018.

68. Serin Y and Acar Tek N: Effect of circadian rhythm on metabolic processes and the regulation of energy balance. Ann Nutr Metab 74: 322-330, 2019.

69. Lemmer B and Oster H: The role of circadian rhythms in the hypertension of diabetes mellitus and the metabolic syndrome. Curr Hypertens Rep 20: 43, 2018.

70. Di R, Luo Q, Mathew D and Bhatwadekar AD: Diabetes alters diurnal rhythm of electroretinogram in $\mathrm{db} / \mathrm{db}$ mice. Yale J Biol Med 92: 155-167, 2019.

71. Wang Q, Tikhonenko M, Bozack SN, Lydic TA, Yan L, Panchy NL, McSorley KM, Faber MS, Yan Y, Boulton ME, et al: Changes in the daily rhythm of lipid metabolism in the diabetic retina. PLoS One 9: e95028, 2014.

72. Luo Q, Xiao Y, Alex A, Cummins TR and Bhatwadekar AD: The diurnal rhythm of insulin receptor substrate-1 (IRS-1) and Kir4.1 in diabetes: Implications for a clock gene Bmal1. Invest Ophthalmol Vis Sci 60: 1928-1936, 2019.

73. Wang Y, An H, Liu T, Qin C, Sesaki H, Guo S, Radovick S, Hussain M, Maheshwari A, Wondisford FE, et al: Metformin improves mitochondrial respiratory activity through activation of AMPK. Cell Rep 29: 1511-1523.e5, 2019. 
74. Alex A, Luo Q, Mathew D, Di R and Bhatwadekar AD Metformin corrects abnormal circadian rhythm and Kir4.1 channels in diabetes. Invest Ophthalmol Vis Sci 61: 46 , 2020.

75. Schultze SM, Hemmings BA, Niessen M and Tschopp O: PI3K/ AKT, MAPK and AMPK signalling: Protein kinases in glucose homeostasis. Expert Rev Mol Med 14: e1, 2012.

76. Lechner J, O'Leary OE and Stitt AW: The pathology associated with diabetic retinopathy. Vision Res 139: 7-14, 2017.

77. Stefanini FR, Badaró E, Falabella P, Koss M, Farah ME and Maia M: Anti-VEGF for the management of diabetic macular edema. J Immunol Res 2014: 632307, 2014.

78. Lai TW, Zhang S and Wang YT: Excitotoxicity and stroke: Identifying novel targets for neuroprotection. Prog Neurobiol 115: $157-188,2014$

79. Welters A, Klüppel C, Mrugala J, Wörmeyer L, Meissner T, Mayatepek E, Heiss C, Eberhard D and Lammert E: NMDAR antagonists for the treatment of diabetes mellitus-Current status and future directions. Diabetes Obes Metab 19 (Suppl 1): S95-S106, 2017.

80. Bai N, Aida T, Yanagisawa M, Katou S, Sakimura K, Mishina M and Tanaka K: NMDA receptor subunits have different roles in NMDA-induced neurotoxicity in the retina. Mol Brain 6: 34 2013.

81. Fuwa M, Kageyama M, Ohashi K, Sasaoka M, Sato R, Tanaka M and Tashiro K: Nafamostat and sepimostat identified as novel neuroprotective agents via NR2B N-methyl-D-aspartate receptor antagonism using a rat retinal excitotoxicity model. Sci Rep 9: 20409, 2019.

82. Han N, Yu L, Song Z, Luo L and Wu Y: Agmatine protects Müller cells from high-concentration glucose-induced cell damage via N-methyl-D-aspartic acid receptor inhibition. Mol Med Rep 12: 1098-1106, 2015.

83. Ozaki H, Inoue R, Matsushima T, Sasahara M, Hayashi A and Mori H: Serine racemase deletion attenuates neurodegeneration and microvascular damage in diabetic retinopathy. PLoS One 13: e0190864, 2018

84. Chen H, Ji Y, Yan X, Su G, Chen L and Xiao J: Berberine attenuates apoptosis in rat retinal Müller cells stimulated with high glucose via enhancing autophagy and the AMPK/mTOR signaling. Biomed Pharmacother 108: 1201-1207, 2018.
85. Barialai L, Strecker MI, Luger AL, Jäger M, Bruns I, Sittig ACM, Mildenberger IC, Heller SM, Delaidelli A, Lorenz NI, et al: AMPK activation protects astrocytes from hypoxia-induced cell death. Int J Mol Med 45: 1385-1396, 2020.

86. Ulbricht E, Pannicke T, Hollborn M, Raap M, Goczalik I, Iandiev I, Härtig W, Uhlmann S, Wiedemann P, Reichenbach A, et al: Proliferative gliosis causes mislocation and inactivation of inwardly rectifying $\mathrm{K}(+)$ (Kir) channels in rabbit retinal glial cells. Exp Eye Res 86: 305-313, 2008.

87. Sene A, Tadayoni R, Pannicke T, Wurm A, El Mathari B, Benard R, Roux MJ, Yaffe D, Mornet D, Reichenbach A, et al: Functional implication of Dp71 in osmoregulation and vascular permeability of the retina. PLoS One 4: e7329, 2009.

88. Vacca O, Charles-Messance H, El Mathari B, Sene A, Barbe P, Fouquet S, Aragón J, Darche M, Giocanti-Aurégan A, Paques M, et al: AAV-mediated gene therapy in Dystrophin-Dp71 deficient mouse leads to blood-retinal barrier restoration and oedema reabsorption. Hum Mol Genet 25: 3070-3079, 2016.

89. Siqueiros-Marquez L, Bénard R, Vacca O, Charles-Messance H, Bolaños-Jimenez R, Guilloneau X, Sennlaub F, Montañez C, Sahel JA, Rendon A, et al: Protection of glial Müller cells by dexamethasone in a mouse model of surgically induced blood-retinal barrier breakdown. Invest Ophthalmol Vis Sci 58: 876-886, 2017.

90. Liu XQ, Kobayashi H, Jin ZB, Wada A and Nao IN: Differential expression of Kir4.1 and aquaporin 4 in the retina from endotoxin-induced uveitis rat. Mol Vis 13: 309-317, 2007.

91. Sun W, Li T, Ma H, Lin S, Xie M, Luo Y, Tian R and Tang S: The effect of $\mathrm{K}^{+}$channel opener pinacidil on the transmembrane potassi channel protein Kir4.1 of retinal Müller cells in vitro and diabetic rats. Panminerva Med 62: 268-270, 2020.

92. Jung E and Kim J: Aloin inhibits Müller cells swelling in a rat model of thioacetamide-induced hepatic retinopathy. Molecules 23: 2806, 2018.

This work is licensed under a Creative Commons Attribution-NonCommercial-NoDerivatives 4.0 International (CC BY-NC-ND 4.0) License. 